\title{
Tachyons in an Expanding Space-Time
}

\author{
ROMAN TOMASCHITZ \\ Department of Physics, Hiroshima University, 1-3-1 Kagami-yama, 724 \\ Higashi-Hiroshima, Japan and Inter-University Centre for Astronomy and \\ Astrophysics, Post Bag 4 Ganeshkhind, Pune 411 007, India
}

\begin{abstract}
Superluminal signal transfer is introduced in the context of an absolute frame of reference provided by the galactic background. The receding galaxies constitute a reference frame, a frame of absolute rest, in which the energy of tachyons (faster-than-light particles) can be defined as a positive definite quantity. The theory presented is essentially covariant, but not relativistic. The causality problem of superluminal signal transfer, which arises in relativistic theories, can be completely avoided. Tachyons are studied in a Robertson-Walker universe with linear expansion factor and negatively curved three-space. The tachyonic dynamics is defined, and it is pointed out how tachyonic events appear to observers who are uniformly moving in the frame of absolute rest. The consequences that the space expansion has on tachyons, e.g. redoubling effects, are discussed. (C) 1997 Elsevier Science Ltd
\end{abstract}

\section{INTRODUCTION}

Whenever one attempts to introduce superluminal signal transfer in special relativity, one is confronted with the breakdown of our traditional conception of causality, e.g. [1-4]. In contemporary physics it is usually taken for granted that (i) every effect has a cause, (ii) the cause precedes the effect and (iii) the distinction of cause and effect is absolute and unambiguous (i.e. every observer will come to the same conclusions on what is cause and what is effect).

In fact, whenever two events are connected by a superluminal signal, one can easily find observers connected by a Lorentz boost, for whom the time order of these events is inverted. (If the tachyon moves from $A$ to $B$ in the rest frame of the first observer, it moves from $B$ to $A$ in the second frame.) The two observers will come to different conclusions on what is cause and what is effect, if they take for granted that the cause precedes the effect.

In Refs [5] and [6] a cosmic mechanics of tachyonic signals was introduced, based on the causality principle (i)-(iii) and an absolute space conception. In special relativity space is the void, generated by coordinate axes. In cosmology, however, space is generated by the galactic grid, and we take this grid as the absolute frame of reference.

We say an observer is at absolute rest if he is comoving with the galactic background, which means that he sees the galaxies isotropically receding. In the standard coordinate representation of a Robertson-Walker universe these observers have constant space coordinates. Galaxies themselves are at absolute rest, despite their recession. To an observer in uniform (i.e. geodesic) motion, the galactic background appears anisotropic, Lorentz-contracted and Doppler-shifted. But this appearance is due to the observer's own movement, it is an artifact of his own frame of rest. As for tachyons, we have therefore to ask what they are in the frame of absolute rest, and how they appear to observers in moving frames, very much contrary to relativity principles, according to which all uniformly moving frames share equal physical reality.

In Section 2 we consider a Robertson-Walker cosmology with linear expansion factor and hyperbolic space-like slices. This is a flat, maximally symmetric four-manifold. Therefore there exist 
symmetry transformations of the four-metric analogous to Lorentz boosts, which synchronize clocks of geodesically moving observers with cosmic time. Cosmic time is defined by the galactic expansion, it is the time in the frame of absolute rest.

In this frame tachyons have only a finite lifetime, which may appear to moving observers to be arbitrarily large or small. There are frames in which the tachyon does not emerge at all. The most surprising effect of the expansion is, that the tachyon may appear as redoubled. This can happen if its energy undergoes a sign change in the moving frame. The tachyon will then appear to the moving observer at two different space points at the same time!

Tachyonic energy can be easily defined in the frame of absolute rest as a positive quantity [5,6]. In moving frames one defines energy and momentum by applying a symmetry transformation, regarding energy and momentum formally as a four-vector. In a moving frame, however, energy defined in this way is no longer positive definite. It is an order rather than interaction parameter. Whenever it becomes negative this indicates a velocity inversion, and a change of the time order. A negative energy means that the cosmic time order in the frame of absolute rest appears distorted to the moving observer. This distortion is a consequence of his motion against the galactic background.

In Section 3 we study the causality of a tachyonic communication process on the basis of this absolute space-time conception. We show that this process is in perfect accord with the abovementioned causality principle (i)-(iii). In Section 4 we present our conclusions.

\section{TACHYONIC DYNAMICS IN AN OPEN UNIVERSE}

To become more specific, let us consider a Robertson-Walker cosmology with linear expansion, and negatively curved three-space,

$$
\begin{gathered}
\mathrm{d} s^{2}=-c^{2} \mathrm{~d} \tau^{2}+a^{2}(\tau) \mathrm{d} \sigma^{2}, \\
\mathrm{~d} \sigma^{2}=R^{2} t^{-2}\left(|\mathrm{~d} z|^{2}+\mathrm{d} t^{2}\right), a(\tau)=c R^{-1} \tau, 0<\tau<\infty .
\end{gathered}
$$

We use the half-space model $H^{3}$ of hyperbolic space $\left(z=x_{1}+i x_{2}, t>0\right.$, cf. Ref. [7]). For the geodesic world-lines of particles $(\varepsilon=1)$ and tachyons $(\varepsilon=-1)$ we have, cf. Ref. [6],

$$
\dot{\tau}^{2}-\tau^{2} t^{-2} \dot{t}^{2}=\varepsilon, T^{2} t^{-1} \dot{t}=: v_{p, t},
$$

the dot denotes differentiation with respect to the curve parameter $s$. We have put here $z=0$, since we consider geodesic motion $(\tau(s), t(s))$ along the $t$-axis of $H^{3}$ (without loss of generality, for $H^{3}$ is homogeneous). $v_{p, t}$ is a real integration constant, and we have put $R=c=1$.

For particles (observers) we obtain

$$
T(s)=\sqrt{s^{2}-v_{p}^{2}}, t(s)=\kappa_{p}\left(s-v_{p}\right)^{1 / 2}\left(s+v_{p}\right)^{-1 / 2},
$$

with $\kappa_{p}$ a positive integration constant. The parameter range is $\infty>s>\left|v_{p}\right|$.

For tachyons we obtain

$$
\tau(s)=\sqrt{v_{t}^{2}-s^{2}}, t(s)=\kappa_{t}\left(v_{t}+s\right)^{1 / 2}\left(v_{t}-s\right)^{-1 / 2},
$$

and

$$
t(\tau)=\kappa_{t}\left(v_{t}-\sqrt{v_{t}^{2}-\tau^{2}}\right)^{1 / 2}\left(v_{t}+\sqrt{v_{t}^{2}-\tau^{2}}\right)^{-1 / 2},
$$

with $\kappa_{t}>0$ and $-\left|v_{t}\right|<s<0$.

The manifold defined by equations (1) and (2) is flat and maximally symmetric [8]; there exist symmetry transformations of the line element (1) analogous to Lorentz boosts. Because we focus 
on geodesic motion along the $t$-axis, it is enough to put $z=0$ in equation (2), and to consider the $(\tau, t)$-plane. As symmetry transformations of the line element (1) we then obtain

$$
\begin{aligned}
& \tau^{\prime}=t^{-1 / 2}(t \tau-a)^{1 / 2}(\tau+b t)^{1 / 2}, \\
& t^{\prime}=t^{1 / 2}(t \tau-a)^{1 / 2}(\tau+b t)^{-1 / 2},
\end{aligned}
$$

with real constants $a$ and $b$.

The inverse of this transformation is obtained by changing the signs of $a$ and $b$. It is important to note, that (7) does not map the whole quadrant $\tau>0, t>0$ onto itself. Only the region

$$
\tau>0, t>0, t \tau-a>0, \tau+b t>0
$$

is mapped onto

$$
\tau^{\prime}>0, t^{\prime}>0, t^{\prime} \tau^{\prime}+a>0, \tau^{\prime}-b t^{\prime}>0 .
$$

The cosmology defined by (1) and (2) can be isometrically mapped onto the interior of the forward light cone, and the transformation (7) corresponds to a space-time shift in Minkowski space, cf. Ref. [8].

To obtain the rest frame $\left(\tau^{\prime}, t^{\prime}\right)$ of an observer defined by integration constants $\left(v_{p}, \kappa_{p}\right)$ in equation (4), we specify

$$
a=-v_{p} \kappa_{p}, b=-v_{p} \kappa_{p}^{-1} .
$$

We have then $\tau^{\prime}=s, t^{\prime}=\kappa_{p}$. So, by means of (7) and (10), one can synchronize a clock in the rest frame $\left(\tau^{\prime}, t^{\prime}\right)$ of a moving observer $\left(v_{p}, \kappa_{p}\right)$ with cosmic time $\tau$ (the time in the frame of absolute rest).

Remark. The rest frame of a geodesically moving observer has the same line element (1) as the frame of absolute rest, though the coordinate range of $\left(\tau^{\prime}, t^{\prime}\right)$ is restricted by equations (9) and (10). But the real difference is that the galactic world-lines, with space coordinates $t(s)=$ const, $z(s)=$ const, in the frame of absolute rest, have non-constant coordinates $\left(t^{\prime}(s), z^{\prime}(s)\right)$ in the rest frame of the moving observer. (In this article we suppress the $z$-coordinates).

In the frame of absolute rest we define tachyonic energy and momentum as

$$
E=m \sqrt{v_{t}^{2} / \tau^{2}-1}, p=m v_{t} \tau^{-2} t(\tau),|p|^{2}=\tau^{2} t^{-2} p^{2},
$$

or by means of the curve parameter $s$,

$$
E=m \dot{\tau}, p=m \dot{t},
$$

with a positive mass $m . E$ and $p$ can also be parametrized by velocity [6].

Remark. It is clear from equations (5) and (11) that tachyons in the frame of absolute rest have only a finite lifetime. Starting their existence at the initial singularity, $\tau=0$, they steadily loose energy until they finally disappear.

Energy and momentum in a moving frame we obtain by formally regarding $(E, p)$ in $(12)$ as a contravariant two-vector, and by transforming it via the differential version of equation (7). Using (5) we obtain

$$
E^{\prime}= \pm \frac{m}{\tau^{\prime}} \sqrt{v_{t}^{\prime 2}-\tau^{\prime 2}}, v_{t}^{\prime}:=v_{t}+\frac{1}{2} \operatorname{sign}\left(v_{t}\right) v_{p}\left(\kappa_{p} / \kappa_{t}-\kappa_{t} / \kappa_{p}\right)
$$

The sign of $E^{\prime}$ depends on whether

$$
\sqrt{v_{t}^{\prime 2}-\tau^{\prime 2}}=\mp\left(s+\frac{1}{2} \operatorname{sign}\left(v_{t}\right) v_{p}\left(\kappa_{p} / \kappa_{t}+\kappa_{t} / \kappa_{p}\right)\right)
$$


(Roots are regarded as positive.)

For the momentum we obtain, if the plus-sign holds true in (13),

$$
p^{\prime}=m v_{t}^{\prime} \tau^{\prime-2} t^{\prime}\left(v_{t}^{\prime}, \tau^{\prime}\right)
$$

with $t^{\prime}\left(v_{t}^{\prime}, \tau^{\prime}\right)$ as in equation (6) (with $v_{t}$ replaced by $v_{t}^{\prime}$, and $\left.\kappa_{t}^{\prime}=\kappa_{t}\right)$. If the energy is negative, we have to replace $t^{\prime}\left(v_{t}^{\prime}, \tau^{\prime}\right)$ by $t^{\prime}\left(-v_{t}^{\prime}, \tau^{\prime}\right)$ in (15).

Remark. We have seen that in this cosmology there exists a natural synchronization of clocks of uniformly moving observers, like in Minkowski space. Only with a well-defined synchronization, valid beyond 'infinitesimal' reasoning in terms of locally geodesic coordinates, can one obtain a well-defined energy in all uniformly moving frames. Note that $(E, p)$, as defined in (12), is not really a vector for tachyons, because the transformations (7) may change the sign of $E$, cf. equation (13). To define energy and momentum in moving frames, we use the frame of absolute rest as a reference frame.

In the following we will study how the trajectory of a tachyon $\left(\kappa_{t}, v_{t}\right)$, cf. equation (5), in the frame of absolute rest $(\tau, t)$ appears to a moving observer with rest frame $\left(\tau^{\prime}, t^{\prime}\right)$ and integration constants $\left(\kappa_{p}, v_{p}\right)$, cf. equation (4). The transformation formulas relating the frames are (7) and (10). Only the part of the tachyon trajectory (5) that satisfies conditions (8) is visible in the moving frame.

We discuss here only one particularly stunning case; a systematic discussion of the qualitative behavior of tachyon trajectories in moving frames is easily possible. We consider $v_{t}>0$. Then the part of the trajectory (5) which is visible in the moving frame [via equations (7) and (10)] is parametrized by

$$
\max \left[-\left(v_{t}+v_{p} \kappa_{p} / \kappa_{t}\right),-v_{t}\right]<s<\min \left[v_{t}-v_{p} \kappa_{t} / \kappa_{p}, 0\right] .
$$

This follows from (8) and the parameter range in equation (5). First of all, it is possible to choose the integration constants in (16) so that no $s$ can satisfy it. The tachyon is then at no time visible in the moving frame. More generally, the lifetime of a tachyon may appear arbitrarily large or small in an appropriately chosen moving frame. Let us now assume that (16) defines a finite range of $s$-values. Then it is important to note that $\tau^{\prime}(s)$, the time component of the tachyon trajectory in the moving frame [namely $\tau^{\prime}(\tau, t)$ in equation (7) with the trajectory (5) inserted], need not be monotonous in this $s$-range. In fact, $\tau^{\prime}(s)$ has a maximum at

$$
s_{\max }=-\frac{1}{2} v_{p}\left(\kappa_{t} / \kappa_{p}+\kappa_{p} / \kappa_{t}\right)
$$

provided $s_{\max }$ lies in the range defined in (16). Also note that $s_{\max }$ is just the value at which the energy (13) changes its sign, cf. equation (14). For $s<s_{\max }, E^{\prime}$ is positive.

Let us choose the integration parameters of observer and tachyon so that $v_{p}>0$ and $v_{t}>v_{p}\left(\kappa_{t} / \kappa_{p}+\kappa_{p} / \kappa_{t}\right)$. Then (16) simply reads as $-v_{t}<s<0$ and $s_{\max }$ as well as $2 s_{\max }$ lie in this range. We have

$$
\tau^{\prime}\left(-v_{t}\right)<\tau^{\prime}\left(2 s_{\max }\right)<\tau^{\prime}\left(s_{\max }\right)>\tau^{\prime}(0), \tau^{\prime}\left(-v_{t}\right)<\tau^{\prime}(0), \tau^{\prime}\left(2 s_{\max }\right)=\tau^{\prime}(0) .
$$

The curve $\tau^{\prime}(s)$ is increasing in $]-v_{t}, s_{\max }[$ and decreasing in $] s_{\max }, 0[$.

The observer $\left(\kappa_{p}, v_{p}\right)$ sees in his frame $\left(\tau^{\prime}, t^{\prime}\right)$ a tachyon $T_{1}^{\prime}$ appearing at time $\tau^{\prime}\left(-v_{t}\right)$, which moves with positive energy. At time $\tau^{\prime}\left(2 s_{\max }\right)\left(=\tau^{\prime}(0)\right)$ a second tachyon $T_{2}^{\prime}$ appears at space point $t^{\prime}(0)$, when $T_{1}^{\prime}$ is at $t^{\prime}\left(2 s_{\max }\right) . T_{2}^{\prime}$ moves with negative energy in $] \tau^{\prime}(0), \tau^{\prime}\left(s_{\max }\right)[$. At $\left(\tau^{\prime}\left(s_{\max }\right), t^{\prime}\left(s_{\max }\right)\right)$ the two tachyons coalesce and disappear with zero energy.

The whole trajectory in the frame of absolute rest is visible for the moving observer. The parameter range $-v_{t}<s<s_{\max }$ corresponds to tachyon $T_{1}^{\prime}$, the range $s_{\max }<s<0$ to tachyon $T_{2}^{\prime}$. Within the time interval $] \tau^{\prime}(0), \tau^{\prime}\left(s_{\max }\right)[$ two tachyons appear in the moving frame, separated in 
space. Tachyon $T_{2}^{\prime}$ appears as moving from $t^{\prime}(0)$ to $t^{\prime}\left(s_{\max }\right)$. In reality, in the frame of absolute rest, a tachyon moves from the initial singularity to $t\left(s_{\max }\right)$ and from $t\left(s_{\max }\right)$ to $t(0)$; the first part of its trajectory appears as $T_{1}^{\prime}$ in the moving frame, the second as $T_{2}^{\prime}$. The time inverted movement of $T_{2}^{\prime}$ is indicated by its negative energy. This is indeed a remarkable phenomenon, for the tachyon in the frame of absolute rest appears in the moving frame at different places at the same time.

\section{TACHYONIC SIGNAL TRANSFER}

Let us finally analyze the causality of a tachyonic communication process. In order to avoid technicalities, and to compare with relativistic theories [2], I consider here a static Minkowski universe. The frame of absolute rest is then distinguished from uniformly moving frames by a static galactic grid. In this frame we define energy and momentum like in equation (12). In moving frames we define energy by means of a Lorentz boost, cf. the remark after equation (15). The following discussion can be carried over, step by step, to the expanding space-time dealt with in Section 2, without any conceptual changes.

$O_{1}$ denotes an observer in the frame of absolute rest $(t, x)$ sitting at $x=0$ and emitting at time $t=0$ a tachyon $T_{1}$ with velocity $v_{1}>1$. A second observer $O_{2}$ (primed coordinates) moves along the $x$-axis with velocity $0<u<1$. At $t=0, O_{2}$ is at $x_{1}>0$. The frames are related by a Lorentz boost as usual. As soon as this tachyon hits $\mathrm{O}_{2}$ (and exchanges energy), $\mathrm{O}_{2}$ responds by emitting a tachyon $T_{2}$ with velocity $v_{2}<-1$. [ $v_{i}$ denote velocities in the frame of absolute rest, and $v_{i}^{\prime}$ in the rest frame of $\mathrm{O}_{2}$. We have $v^{\prime}=(v-u)(1-u v)^{-1}$. In the following it is advisable to have the graph of this function in mind.]

If $1<v_{1}<u^{-1}$, then $v_{1}^{\prime}>1$, and the observer $O_{2}$ sees the tachyon $T_{1}$ emitted by $O_{1}$ and moving toward him with positive energy. All his observations qualitatively agree with those of $O_{1}$. If, however, $v_{1}>u^{-1}$, then the cosmic time order is inverted in the rest frame of $O_{2}$. It appears to $\mathrm{O}_{2}$ that the tachyon is emitted by $\mathrm{O}_{2}$ himself, and moves toward $O_{1}$. The energy of this tachyon is negative in the rest frame of $\mathrm{O}_{2}$, so the emission of the tachyon he will notice as a gain of energy, and $O_{1}$ will appear to loose energy by absorbing $T_{1}$. The gain and loss of energy have the same time order as in the frame of absolute rest. But the fact that the tachyon appears to move from $O_{2}$ to $O_{1}$ is an illusion of the rest frame of $O_{2}$, like the Lorentz contraction of the galactic background. It is an artifact of the movement of $\mathrm{O}_{2}$ in the frame of absolute rest.

Anyway, as soon as an energy exchange occurs between $T_{1}$ and $O_{2}, O_{2}$ emits a tachyon $T_{2}$ carrying his response. If the velocity $v_{2}^{\prime}$ of $T_{2}$ is within the range ] $-u^{-1},-1$, then $T_{2}$ hits $O_{1}$ at a time $t>0$. The energy of $T_{2}$ is positive in the rest frame of $O_{2}$, and the observations of $O_{1}$ and $\mathrm{O}_{2}$ agree qualitatively. But if $v_{2}^{\prime}<-u^{-1}$, the two observations differ crucially. Observer $O_{1}$ in the frame of absolute rest sees $T_{2}$ emitted by $O_{2}$ and moving away from him (namely $O_{1}$ ) with positive velocity $v_{2} . T_{2}$ can never reach $O_{1}$. In the rest frame of $O_{2}$, however, the tachyon has negative energy, a velocity inversion takes place, and it appears to $O_{2}$ that $T_{2}$ indeed collides with $O_{1}$.

In the moving frame the indefinite energy is an order parameter. A negative energy always indicates to observer $\mathrm{O}_{2}$ that the cosmic reality is distorted in his frame. By means of a Lorentz transformation, whose parameters he can read off from the galactic background passing by, he can inquire on the physical process actually taking place in the frame of absolute rest.

Causality is preserved in this communication process, since under no circumstances the tachyon $T_{2}$ can influence the past $t<0$ of observer $O_{1}$. The response $T_{2}$ cannot arrive before $T_{1}$ is emitted. This is very contrary to the relativistic interpretation of this process [2], which is based on the hypothesis that the observations of $O_{1}$ and $O_{2}$ are equally real, and on a reinterpretation of negative energies like in relativistic quantum mechanics [1]. Relativistic tachyonic theories cannot be reconciled with the traditional causality principle (i)-(iii) as stated in Section 1, see Ref. [6] for a complete discussion. 


\section{CONCLUDING REMARKS}

The absolute space-time conception in this cosmic context is not in conflict with any experimental result. The formalism we use to describe tachyons is essentially covariant and classical. In particular, we are not forced to give up the causality principle, which would be a strange thing to do in a theory based on classical deterministic mechanics. Tachyonic energy is defined as a positive definite interaction parameter in the frame of absolute rest. Energy is an order parameter in moving frames, indicating time inversion. Accordingly, there is no reinterpretation of negative energies necessary. Interactions of particles and tachyons can be studied in the frame of absolute rest in terms of elastic head-on collisions and energy-momentum conservation [6].

I should finally also mention my reason to speculate on the existence of superluminal particles. It is just very tempting to assume this in a cosmology with an open three-space. A uniform bound on the speed of signal transfer does not really make sense in an infinite universe.

Acknowledgements - The author acknowledges the support of the Japan Society for the Promotion of Science, Contract No. P-95378, and in particular the stimulating atmosphere of the IUCAA, where most of this work was carried out.

\section{REFERENCES}

1. Bilaniuk, O., Deshpande, V. and Sundarshan, E., Meta-relativity. Am. J. Phys., 1962, 30, 718-723.

2. Feinberg, G., Possibility of faster-than-light particles. Phys. Rev., 1967, 159, 1089-1105.

3. Recami, E., Classical tachyons and possible applications. Riv. Nuovo Cimento, 1986, 9, 1-178.

4. El Naschie, M. S., On conjugate complex time. Chaos, Solitons \& Fractals, 1995, 5, 1551-1555.

5. Tomaschitz, R., Topological deformations, chaos, and tachyons in cosmology. In Proceedings of the 20th Colloquium on Group Theoretical Methods in Physics, Osaka, Japan, eds A. Arima et al.. World Scientific, Singapore, pp. 111-124.

6. Tomaschitz, R., Tachyonic chaos and causality in the open universe. Chaos, Solitons \& Fractals, 1996, 7, 753-768.

7. Tomaschitz, R. , Classical and quantum dispersion in Robertson-Walker cosmologies. J. Math. Phys., 1993, 34, 1022-1042.

8. Infeld, L. and Schild, A., A new approach to kinematic cosmology. Phys. Rev., 1945, 68, $250-272$. 